\title{
@creative
commons
}

Also available at http://amc-journal.eu

ISSN 1855-3966 (printed edn.), ISSN 1855-3974 (electronic edn.)

ARS MATHEMATICA CONTEMPORANEA 10 (2016) 255-268

\section{Iterated claws have real-rooted genus polynomials}

\author{
Jonathan L. Gross * \\ Department of Computer Science, Columbia University, New York, NY 10027, USA
}

Toufik Mansour

Department of Mathematics, University of Haifa, 3498838 Haifa, Israel

Thomas W. Tucker ${ }^{\dagger}$

Department of Mathematics, Colgate University, Hamilton, NY 13346, USA

David G. L. Wang $\ddagger$
School of Mathematics and Statistics, Beijing Institute of Technology, 102488 Beijing, P.
R. China

Received 10 September 2013, accepted 27 September 2015, published online 30 November 2015

\begin{abstract}
We prove that the genus polynomials of the graphs called iterated claws are real-rooted. This continues our work directed toward the 25-year-old conjecture that the genus distribution of every graph is log-concave. We have previously established log-concavity for sequences of graphs constructed by iterative vertex-amalgamation or iterative edgeamalgamation of graphs that satisfy a commonly observable condition on their partitioned genus distributions, even though it had been proved previously that iterative amalgamation does not always preserve real-rootedness of the genus polynomial of the iterated graph. In this paper, the iterated topological operation is adding a claw, rather than vertex- or edge-amalgamation. Our analysis here illustrates some advantages of employing a matrix representation of the transposition of a set of productions.
\end{abstract}

Keywords: Topological graph theory, graph genus polynomials, log-concavity, real-rootedness.

Math. Subj. Class.: 05A15, 05A20, 05C10

* Supported by Simons Foundation Grant \#315001.

${ }^{\dagger}$ Supported by Simons Foundation Grant \#317689.

${ }^{\ddagger}$ Supported by National Natural Science Foundation of China Grant No. 11101010

E-mail addresses: gross@cs.columbia.edu (Jonathan L. Gross), tmansour@univ.haifa.ac.il (Toufik Mansour), ttucker@colgate.edu (Thomas W. Tucker), david.combin@gmail.com; glw@bit.edu.cn (David G. L. Wang)

(a) (i) This work is licensed under http://creativecommons.org/licenses/by/3.0/ 


\section{Introduction}

Graphs are implicitly taken to be connected. Our graph embeddings are cellular and orientable. For general background in topological graph theory, see [1,9]. Prior acquaintance with the concepts of partitioned genus distribution (abbreviated here as pgd) and production (e.g., see $[5,11]$ ) is prerequisite to reading this paper. Subject to this prerequisite, the exposition here is intended to be accessible both to graph theorists and to combinatorialists.

The genus distribution of a graph $G$ is the sequence $g_{0}(G), g_{1}(G), g_{2}(G), \ldots$, where $g_{i}(G)$ is the number of combinatorially distinct embeddings of $G$ in the orientable surface of genus $i$. A genus distribution contains only finitely many positive numbers, and there are no zeros between the first and last positive numbers. The genus polynomial is the polynomial

$$
\Gamma_{G}(z)=g_{0}(G)+g_{1}(G) z+g_{2}(G) z^{2}+\ldots
$$

We say that a sequence $A=\left(a_{k}\right)_{k=0}^{n}$ is nonnegative if $a_{k} \geq 0$ for all $k$. An element $a_{k}$ is said to be an internal zero of $A$ if there exist indices $i$ and $j$ with $i<k<j$, such that $a_{i} a_{j} \neq 0$ and $a_{k}=0$. If $a_{k-1} a_{k+1} \leq a_{k}^{2}$ for all $k$, then $A$ is said to be log-concave. If there exists an index $h$ with $0 \leq h \leq n$ such that

$$
a_{0} \leq a_{1} \leq \cdots \leq a_{h-1} \leq a_{h} \geq a_{h+1} \geq \cdots \geq a_{n}
$$

then $A$ is said to be unimodal. It is well-known that any nonnegative log-concave sequence without internal zeros is unimodal, and that any nonnegative unimodal sequence has no internal zeros. A prior paper [7] by the present authors provides additional contextual information regarding log-concavity and genus distributions.

\subsection{The LCGD Conjecture and Real-Rootedness Problems}

For convenience, we sometimes abbreviate the phrase "log-concave genus distribution" as $\boldsymbol{L C G D}$. Proofs that closed-end ladders and doubled paths have LCGDs [2] were based on closed formulas for their genus distributions. Proof that bouquets have LCGDs [8] was based on a recursion. The following conjecture was formulated in [8]:

LCGD Conjecture: Every graph has a log-concave genus distribution.

Stahl [12] used the term " $H$-linear" to describe chains of graphs obtained by amalgamating copies of a fixed graph $H$. He conjectured that a number of " $H$-linear" families of graphs have genus polynomials with nonpositive real roots, which implies the logconcavity of their sequences of coefficients, by Newton's theorem. (Since all the coefficients of a genus polynomial are non-negative, it follows that all the roots are non-positive.) Although it was shown [14] that the genus polynomials of some such families do indeed have real roots, Stahl's conjecture of real-rootedness for $W_{4}$-linear graphs (where $W_{4}$ is the 4-wheel) was disproved by Liu and Wang [10].

Our previous paper [7] proves, nonetheless, that the genus distribution of every graph in the $W_{4}$-linear sequence is log-concave. Thus, even though Stahl's proposed approach to log-concavity via roots of genus polynomials is sometimes infeasible, [7] does support Stahl's expectation that chains of copies of a graph are a relatively accessible aspect of the general LCGD problem. Moreover, Wagner [14] has proved the real-rootedness of the genus polynomials for a number of graph families for which Stahl made specific conjectures of real-rootedness. 
This leads to a couple of research problems that are subordinate to the LCGD Conjecture, as follows:

Real-rootedness Problem: Characterize the graphs whose genus polynomials are not real-rooted.

Real-rootedness Chain Problem: Characterize the graphs $H$ whose genus polynomials are real-rooted but whose $H$-linear chains contain graphs whose genus polynomials are not real-rooted.

Furthermore, we shall see here that Stahl's method of representing what we have elsewhere $([4,6])$ presented as a transposition of a production system for a surgical operation on graph embeddings as a matrix of polynomials can simplify a proof that a family of graphs has log-concave genus distributions.

\subsection{Interlacing Roots in a Genus Polynomial Sequence}

The earliest proofs $[2,8]$ of the log-concavity of the genus polynomials for a sequence of graphs appealed directly to the condition $a_{j-1} a_{j+1} \leq a_{j}^{2}$. The need for more powerful techniques motivated the development of the linear combination techniques of [7]. Here, to prove the log-concavity of the genus polynomials for the sequence of iterated claws, we combine Newton's theorem that a real-rooted polynomial is log-concave (Theorem 4.1) with a focus on interlacing of roots of consecutive genus polynomials for the graphs in the sequence to prove their log-concavity.

\section{The Sequence of Iterated Claws}

Let the rooted graph $\left(Y_{0}, u_{0}\right)$ be isomorphic to the dipole $D_{3}$, and let the root $u_{0}$ be either vertex of $D_{3}$. For $n=1,2, \ldots$, we define the iterated claw $\left(Y_{n}, u_{n}\right)$ to be the graph obtained the following surgical operation:

Newclaw: Subdivide each of the three edges incident on the root vertex $u_{n-1}$ of the iterated claw $\left(Y_{n-1}, u_{n-1}\right)$, and then join the three new vertices obtained thereby to a new root vertex $u_{n}$.

Figure 1 illustrates the graph $\left(Y_{3}, u_{3}\right)$.

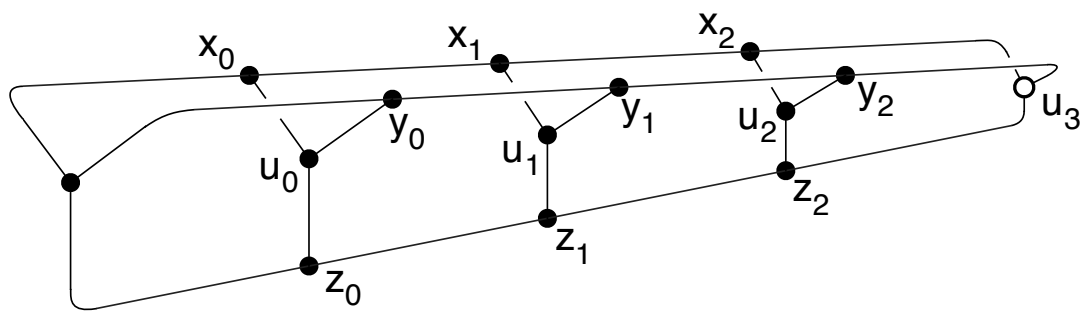

Figure 1: The rooted graph $\left(Y_{3}, u_{3}\right)$.

The graph $K_{1,3}$ is commonly called a claw graph, which accounts for our name iterated claw. The notation $Y_{n}$ reflects the fact that a claw graph looks like the letter $Y$. We observe 
that $Y_{1} \cong K_{3,3}$. A recursion for the genus distribution of the iterated claw graphs is derived in [6]. We observe that, whereas all of Stahl's examples [12] of graphs with log-concave genus distributions are planar, the sequence of iterated claws has rising minimum genus. (Example 3.2 of [7] is another sequence of rising minimum genus. However, the graphs in that sequence have cutpoints, unlike the iterated claws.)

We have seen in previous studies of genus distribution (especially [3]) that the number of productions and simultaneous recursions rises rapidly with the number of roots and the valences of the roots. The surgical operation newclaw is designed to circumvent this problem.

For a single-rooted iterated claw $\left(Y_{n}, u_{n}\right)$, we can define three partial genus distributions, also called partials. Let

$$
\begin{aligned}
& a_{n, i}=\text { the number of embeddings } Y_{n} \rightarrow S_{i} \text { such that } \\
& \text { three different } \mathrm{fb} \text {-walks are incident on the root } u_{n} \text {; } \\
& b_{n, i}=\text { the number of embeddings } Y_{n} \rightarrow S_{i} \text { such that exactly } \\
& \text { two different fb-walks are incident on the root } u_{n} \text {; } \\
& c_{n, i}=\text { the number of embeddings } Y_{n} \rightarrow S_{i} \text { such that } \\
& \text { one fb-walk is incident three times on the root } u_{n} \text {. }
\end{aligned}
$$

We also define partial genus polynomials to be the generating functions

$$
\begin{aligned}
& A_{n}(z)=\sum_{i=0}^{\infty} a_{n, i} z^{i} \\
& B_{n}(z)=\sum_{i=0}^{\infty} b_{n, i} z^{i} \\
& C_{n}(z)=\sum_{i=0}^{\infty} c_{n, i} z^{i} .
\end{aligned}
$$

Clearly, the full genus distribution is the sum of the partials. That is, for $i=0,1,2, \ldots$, we have

and

$$
g_{i}\left(Y_{n}\right)=a_{n, i}+b_{n, i}+c_{n, i}
$$

$$
\Gamma_{Y_{n}}(z)=A_{n}(z)+B_{n}(z)+C_{n}(z)
$$

We define $g_{n, i}=g_{i}\left(Y_{n}\right)$.

Remark 2.1. Partitioned genus distributions and recursion systems for pgds were first used by Furst, Gross, and Statman [2]. Stahl [12] was first to employ a matrix equivalent of a production system to investigate log-concavity.

Theorem 2.2. For $n>1$, the effect on the pgd of applying the operation newclaw to the iterated claw $\left(Y_{n-1}, u_{n-1}\right)$ corresponds to the following system of three productions:

$$
\begin{aligned}
& a_{i} \longrightarrow \quad 12 b_{i+1}+4 c_{i+2} \\
& b_{i} \longrightarrow 2 a_{i}+12 b_{i+1}+2 c_{i+1} \\
& c_{i} \longrightarrow 8 a_{i}+8 c_{i+1}
\end{aligned}
$$


Proof. This is Theorem 4.5 of [6].

Corollary 2.3. For $n>1$, the effect on the pgd of applying the operation newclaw to the iterated claw $\left(Y_{n-1}, u_{n-1}\right)$ corresponds to the following recurrence relations:

$$
\begin{aligned}
& a_{n, i}= \\
& b_{n, i}=12 b_{n-1, i}+8 c_{n-1, i} \\
& c_{n, i}=4 a_{n-1, i-2}+2 b_{n-1, i-1}
\end{aligned}
$$

Proof. The recurrence system (2.4), (2.5), (2.6) is induced by the production system (2.1), (2.2), (2.3).

It is convenient to express such a recurrence system in matrix form:

$$
V\left(Y_{n}\right)=M(z) \cdot V\left(Y_{n-1}\right)
$$

with the production matrix

$$
M(z)=\left[\begin{array}{ccc}
0 & 2 & 8 \\
12 z & 12 z & 0 \\
4 z^{2} & 2 z & 8 z
\end{array}\right] .
$$

Since the initial graph $Y_{0}$ in the sequence of iterated claws is isomorphic to the dipole $D_{3}$, the initial column vector for the sequence $V\left(Y_{n}\right)$ is

$$
V\left(Y_{0}\right)=\left[\begin{array}{l}
A_{0}(z) \\
B_{0}(z) \\
C_{0}(z)
\end{array}\right]=\left[\begin{array}{c}
2 \\
0 \\
2 z
\end{array}\right]
$$

Proposition 2.4. The column vector $V\left(Y_{n}\right)$ is the product of the matrix power $M^{n}(z)$ with the column vector $V\left(Y_{0}\right)$.

Corollary 2.5. The column vector $V\left(Y_{n}\right)$ is the product of the matrix power $M^{n+1}(z)$ with the (artificially labeled) column vector

$$
V\left(Y_{-1}\right)=\left(\begin{array}{c}
0 \\
0 \\
1 / 4
\end{array}\right)
$$

Corollary 2.6. To prove that every iterated claw has an LCGD, it is sufficient to prove that the sum of the third column of the matrix $M^{n}(z)$ is a log-concave polynomial.

\section{Characterizing Genus Polynomials for Iterated Claws}

In this section, we investigate some properties of the genus polynomials of iterated claws. Corollary 2.6 leads us to focus on the sum of the third column of the matrix $M^{n}(z)$, which is expressible as $(1,1,1) M^{n}(z)\left(4 V\left(Y_{-1}\right)\right)$, which implies that it equals 4 times the genus polynomial of the iterated claw $Y_{n-1}$. Theorem 3.1 formulates a generating function $f(z, t)$ for this sequence of sums, and Theorem 3.2 uses the generating function to construct an expression for the genus polynomials from which we establish interlacing of roots in Section 4. 
Theorem 3.1. The generating function $f(z, t)=\sum_{n \geq 0}(1,1,1) M^{n}(z)\left(4 V\left(Y_{-1}\right)\right) t^{n}$ for the sequence of sums of the third column of $M^{n}(z)$ has the closed form

$$
f(z, t)=\frac{1+(8-12 z) t-24 z t^{2}}{1-20 z t+8 z(8 z-3) t^{2}+384 z^{3} t^{3}} .
$$

Proof. Let $\left(p_{n}, q_{n}, r_{n}\right)=(1,1,1) M^{n}(z)$ for all $n \geq 0$. Then

$$
\begin{aligned}
\left(p_{n+1}, q_{n+1}, r_{n+1}\right) & =\left(p_{n}, q_{n}, r_{n}\right) M(z) \\
& =\left(12 z q_{n}+4 z^{2} r_{n}, 2 p_{n}+12 z q_{n}+2 z r_{n}, 8 p_{n}+8 z r_{n}\right) .
\end{aligned}
$$

The third coordinate of Equation (3.2) implies that

$$
p_{n}=\frac{1}{8}\left(r_{n+1}-8 z r_{n}\right)
$$

By combining (3.3) with the first coordinate of (3.2) we obtain

$$
q_{n}=\frac{1}{96 z}\left(r_{n+2}-8 z r_{n+1}-32 z^{2} r_{n}\right)
$$

The second coordinate of (3.2) yields

$$
q_{n+1}=2 p_{n}+12 z q_{n}+2 z r_{n}
$$

Substituting (3.3) and (3.4) (twice) into (3.5) leads to the recurrence relation

$$
r_{n}=20 z r_{n-1}+8 z(3-8 z) r_{n-2}-384 z^{3} r_{n-3}
$$

with

$$
\begin{aligned}
& r_{0}=1, \\
& r_{1}=8+8 z, \\
& r_{2}=160 z+96 z^{2} .
\end{aligned}
$$

By multiplying Recurrence (3.6) by $t^{n}$ and summing over all $n \geq 0$, we obtain Generating Function (3.1).

It is easy to see that $\Gamma_{Y_{n}}(z)=r_{n+1} / 4$, where $r_{n}$ is defined in the proof of Theorem 3.1. In terms of $\Gamma_{Y_{n}}(z)$, the recurrence relation (3.6) becomes

$$
\Gamma_{Y_{n}}(z)=20 z \Gamma_{Y_{n-1}}(z)+8 z(3-8 z) \Gamma_{Y_{n-2}}(z)-384 z^{3} \Gamma_{Y_{n-3}}(z) .
$$

Theorem 3.2 provides an explicit expression for the genus polynomial $\Gamma_{Y_{n}}(z)$, a result is of independent interest. It is not used here toward proof of log-concavity.

Theorem 3.2. The genus polynomial of the iterated claw $Y_{n}$ is given by where

$$
(1,1,1) M^{n+1}(z) V\left(Y_{-1}\right)=2^{n-1}\left(h_{n+1}(z)+2(2-3 z) h_{n}(z)-6 z h_{n-1}(z)\right),
$$

$$
h_{n}(z)=\sum_{2 j+i_{1}+i_{2}+i_{3}=n}\left(\begin{array}{c}
j+i_{1} \\
i_{1}
\end{array}\right)\left(\begin{array}{c}
j+i_{2} \\
i_{2}
\end{array}\right)\left(\begin{array}{c}
j+i_{3} \\
i_{3}
\end{array}\right)(1+\sqrt{3})^{i_{2}}(1-\sqrt{3})^{i_{3}} 3^{j+i_{1}}(2 z)^{n-j} .
$$


Proof. By Theorem 3.1, we have

$$
f(z, t)=\sum_{n \geq 0}(1,1,1) M^{n}\left(4 V\left(Y_{0}\right)\right) t^{n}=\frac{1+(8-12 z) t-24 z t^{2}}{1-20 z t+8 z(8 z-3) t^{2}+384 z^{3} t^{3}} .
$$

Thus,

$$
\begin{aligned}
f(z / 2, t / 2) & =\frac{1+(4-3 z) t-3 z t^{2}}{1-5 z t+z(4 z-3) t^{2}+6 z^{3} t^{3}} \\
& =\frac{1+(4-3 z) t-3 z t^{2}}{\left(1-2 z t-2 z^{2} t^{2}\right)(1-3 z t)-3 z t^{2}} \\
& =\sum_{j \geq 0} \frac{\left(1+(4-3 z) t-3 z t^{2}\right) 3^{j} z^{j} t^{2 j}}{(1-3 z t)^{j+1}(1+\sqrt{3} z t)^{j+1}(1-\sqrt{3} z t)^{j+1}} .
\end{aligned}
$$

Using the combinatorial identity $(1-a t)^{-m}=\sum_{j \geq 0}\left(\begin{array}{c}m-1+j \\ j\end{array}\right) a^{j} t^{j}$, and then finding the coefficient of $t^{n}$, we derive the equation

$$
(1,1,1) M^{n}(z / 2) V\left(Y_{0}\right)=2^{n-2}\left(h_{n}(z)+2(2-3 z) h_{n-1}(z)-6 z h_{n-2}(z)\right),
$$

which, by Corollary 2.5, completes the proof.

Now let $g_{n, i}$ be the coefficient of $z^{i}$ in $\Gamma_{Y_{n}}(z)$. The following table of values of $g_{n, i}$ for $n \leq 4$ is derived in [6].

\begin{tabular}{|c|cccccc|}
\hline$g_{n, i}$ & $i=0$ & 1 & 2 & 3 & 4 & 5 \\
\hline$n=0$ & 2 & 2 & 0 & 0 & 0 & 0 \\
1 & 0 & 40 & 24 & 0 & 0 & 0 \\
2 & 0 & 48 & 720 & 256 & 0 & 0 \\
3 & 0 & 0 & 1920 & 11648 & 2816 & 0 \\
4 & 0 & 0 & 1152 & 52608 & 177664 & 30720 \\
\hline
\end{tabular}

Denote by $\mathcal{P}_{s, t}$ the set of polynomials of the form $\sum_{k=s}^{t} a_{k} z^{k}$, where $a_{k}$ is a positive integer for any $s \leq k \leq t$. The above table suggests that $\Gamma_{Y_{n}}(z) \in \mathcal{P}_{\lfloor(n+1) / 2\rfloor, n+1}$ for $n \leq 4$. Theorem 3.3 shows that it holds true in general. Like Theorem 3.2, this enumerative result is of independent interest and is not used toward proof of log-concavity.

Theorem 3.3. For all $n \geq 0$, the polynomial $\Gamma_{Y_{n}}(z) \in \mathcal{P}_{\lfloor(n+1) / 2\rfloor, n+1}$. Moreover, we have the leading coefficient

$$
g_{n, n+1}=4^{n} \sum_{k=0}^{\lfloor(n+1) / 2\rfloor}\left(\begin{array}{c}
n+2 \\
2 k+1
\end{array}\right) 3^{k}
$$

and, for any number $i$ such that $\lfloor(n+1) / 2\rfloor+1 \leq i \leq n$, we have

$$
g_{n, i}>11 g_{n-1, i-1}
$$


Proof. We see in the table above, for $n \leq 4$, that $\gamma_{\min }\left(Y_{n}\right)=\lfloor(n+1) / 2\rfloor$ and that $\gamma_{\max }\left(Y_{n}\right)=n+1$, or equivalently, that $\Gamma_{Y_{n}}(z) \in \mathcal{P}_{\lfloor(n+1) / 2\rfloor, n+1}$. We see also, for $n \leq 4$, that Equation (3.9) and Inequality (3.10) are true. Now suppose that $n \geq 5$. For convenience, let $g_{k, i}=0$ for all $i<0$. We can also take $g_{k, i}=0$ for $i>k+1$, by induction using (3.8), for $k<n$. From Recurrence (3.8) and the induction hypothesis, we have

$$
g_{n, i}=20 g_{n-1, i-1}+24 g_{n-2, i-1}-64 g_{n-2, i-2}-384 g_{n-3, i-3}, \quad n \geq 3 .
$$

For $i>n+1$, the induction hypothesis implies that each of the four terms on the right side of Recurrence (3.11) is zero-valued. So the degree of $\Gamma_{Y_{n}}(z)$ is at most $n+1$. Let $s_{i}=g_{i, i+1}$. Taking $i=n+1$ in (3.11), we get

$$
s_{n}=20 s_{n-1}-64 s_{n-2}-384 s_{n-3},
$$

with the initial values $s_{0}=2, s_{1}=24, s_{2}=256$. The above recurrence can be solved by a standard generating function method, see [15, p.8]. In practice, we use the command rsolve in the software Maple and get the explicit formula directly as

$$
s_{n}=4^{n} \sum_{k \geq 0}\left(\begin{array}{c}
n+2 \\
2 k+1
\end{array}\right) 3^{k} .
$$

It follows that $g_{n, n+1}>0$. Hence the degree of $\Gamma_{Y_{n}}(z)$ is exactly $n+1$.

Similarly, for $i<\lfloor(n+1) / 2\rfloor$, the four terms on the right side of (3.11) are zerovalued, so the minimum genus of $Y_{n}$ is at least $\lfloor(n+1) / 2\rfloor$. Moreover, applying (3.11) with $i=\lfloor(n+1) / 2\rfloor$ and using the induction hypothesis $g_{k, i}=0$ for all $i<\lfloor(k+1) / 2\rfloor$ with $k<n$, we find the first term is positive for $n$ odd and zero for $n$ even, the second term is always positive, and the third and fourth terms are always zero. In other words,

$$
g_{n,\lfloor(n+1) / 2\rfloor}=20 g_{n-1,\lfloor(n+1) / 2\rfloor-1}+24 g_{n-2,\lfloor(n+1) / 2\rfloor-1} \geq 24 g_{n-2,\lfloor(n+1) / 2\rfloor-1}>0 .
$$

This confirms the minimum genus of $Y_{n}$ is exactly $\lfloor(n+1) / 2\rfloor$.

Now consider $i$ such that $\lfloor(n+1) / 2\rfloor+1 \leq i \leq n$. By (3.11), and using (3.10) inductively, we deduce

$$
\begin{aligned}
g_{n, i} & =11 g_{n-1, i-1}+24 g_{n-2, i-1}+\left(9 g_{n-1, i-1}-64 g_{n-2, i-2}-384 g_{n-3, i-3}\right) \\
& >11 g_{n-1, i-1}+24 g_{n-2, i-1}+\left(35 g_{n-2, i-2}-384 g_{n-3, i-3}\right) \\
& >11 g_{n-1, i-1}+24 g_{n-2, i-1}+g_{n-3, i-3} \\
& \geq 11 g_{n-1, i-1} .
\end{aligned}
$$

So Inequality (3.10) holds true. It follows that $g_{n, i}>0$. Hence

$$
\Gamma_{Y_{n}}(z) \in \mathcal{P}_{\lfloor(n+1) / 2\rfloor, n+1}
$$

This completes the proof. 


\section{Genus Polynomials for Iterated Claws are Real-Rooted}

Our goal in this section is to establish in Theorem 4.3 the real-rootedness of the genus polynomials $\Gamma_{Y_{n}}(z)$ of the iterated claws, via an associated sequence $W_{n}(z)$ of normalized polynomials. It follows from this real-rootedness that the genus polynomials for iterated claws are log-concave, by the following theorem of Newton.

Theorem 4.1 (Newton's theorem). Let $a_{0}, a_{1}, \ldots, a_{n}$ be real numbers and let all the roots of the polynomial

$$
P(x)=\sum_{j=0}^{n} a_{i} x^{i}
$$

be real. Then $a_{j}^{2} \geq a_{j-1} a_{j+1}$ for $j=1, \ldots, n-1$.

Proof. For instance, see Theorem 2 of [13].

To proceed, we "normalize" the polynomials $\Gamma_{Y_{n}}(z)$ by defining

$$
W_{n}(z)=z^{-\lfloor(n+1) / 2\rfloor} \Gamma_{Y_{n}}(z),
$$

so that $W_{n}(z)$ starts from a non-zero constant term, and has the same non-zero roots as $\Gamma_{Y_{n}}(z)$. We use the symbol $d_{n}$ to denote the degree of $W_{n}(z)$, that is,

$$
d_{n}=\operatorname{deg} W_{n}(z)=(n+1)-\left\lfloor\frac{n+1}{2}\right\rfloor=\left\lceil\frac{n+1}{2}\right\rceil .
$$

By Theorem 3.3, we have $W_{n}(z) \in \mathcal{P}_{0, d_{n}}$. Substituting (4.1) into the recurrence relation (3.8), we derive

$$
W_{n}(z)= \begin{cases}20 z W_{n-1}(z)+8(3-8 z) W_{n-2}(z)-384 z^{2} W_{n-3}(z), & \text { if } n \text { is even } \\ 20 W_{n-1}(z)+8(3-8 z) W_{n-2}(z)-384 z W_{n-3}(z), & \text { if } n \text { is odd }\end{cases}
$$

with the initial polynomials

$$
\begin{aligned}
& W_{0}(z)=2(1+z) \\
& W_{1}(z)=8(5+3 z) \\
& W_{2}(z)=16\left(3+45 z+16 z^{2}\right) .
\end{aligned}
$$

Let $\mathcal{P}$ denote the union $\cup_{n \geq 0} \mathcal{P}_{0, n}=\cup_{n \geq 0}\left\{\sum_{k=0}^{n} a_{k} z^{k} \mid a_{k} \in \mathbb{Z}^{+}\right\}$. Lemma 4.2 is ultimately a consequence of the intermediate value theorem.

Lemma 4.2. Let $P(x), Q(x) \in \mathcal{P}$. Suppose that $P(x)$ has roots $x_{1}<x_{2}<\cdots<x_{\operatorname{deg} P \text {, }}$

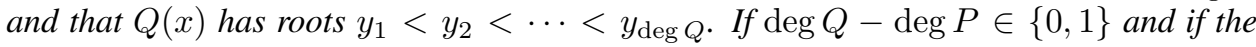
roots interlace so that

$$
x_{1}<y_{1}<x_{2}<y_{2}<\cdots,
$$

then

$$
\begin{aligned}
(-1)^{i+\operatorname{deg} P} P\left(y_{i}\right)>0 & \text { for all } 1 \leq i \leq \operatorname{deg} Q \\
(-1)^{j+\operatorname{deg} Q} Q\left(x_{j}\right)<0 & \text { for all } 1 \leq j \leq \operatorname{deg} P .
\end{aligned}
$$


Proof. Since $P(x)$ is a polynomial with positive coefficients, we have

$$
(-1)^{\operatorname{deg} P} P(-\infty)>0 \text {. }
$$

We suppose first that $\operatorname{deg} P(x)$ is odd, and we consider the curve $P(x)$. We see that Inequality (4.7) reduces to $P(-\infty)<0$. Thus, the curve $P(x)$ starts in the lower half plane and intersects the $x$-axis at its first root, $x_{1}$. From there, the curve $P(x)$ proceeds without going below the $x$-axis, until it meets the second root, $x_{2}$. Since $x_{1}<y_{1}<x_{2}$, we recognize that (4.5) holds for $i=1$, i.e.,

$$
P\left(y_{1}\right)>0
$$

After passing through $x_{2}$, the curve $P(x)$ stays below the $x$-axis up to the third root, $x_{3}$. It is clear that the curve $P(x)$ continues going forward, intersecting the $x$-axis in this alternating way. It follows from this alternation that

$$
P\left(y_{k}\right) P\left(y_{k+1}\right)<0 \quad \text { for all } 1 \leq k \leq \operatorname{deg} Q-1 .
$$

From (4.8) and (4.9), we conclude that (4.5) holds for all $1 \leq i \leq \operatorname{deg} Q$, when $\operatorname{deg} P(x)$ is odd.

We next suppose that $\operatorname{deg} P(x)$ is even. In this case, we can draw the curve $P(x)$ so that it starts in the upper half plane, first intersects the $x$-axis at $x_{1}$, then goes below the axis up to $x_{2}$, and continues alternatingly. Therefore the sign-alternating relation (4.9) still holds. Since $P\left(y_{1}\right)<0$ when $\operatorname{deg} P(x)$ is even, we have proved (4.5).

It is obvious that Inequality (4.6) can be shown along the same line. This completes the proof of Lemma 4.2.

Now we proceed with our main theorem on the genus polynomial of iterated claws. Beyond proving real-rootedness of the genus polynomials, we derive two interlacing relationships on their roots.

Theorem 4.3. For every $n \geq 0$, the polynomial $W_{n}(z)$ is real-rooted. Moreover, if the roots of $W_{k}(z)$ are denoted by $x_{k, 1}<x_{k, 2}<\cdots$, then we have the following interlacing properties:

(i) for every $n \geq 2$, the polynomial $W_{n}(z)$ has one more root than $W_{n-2}(z)$, and the roots interlace so that

$$
x_{n, 1}<x_{n-2,1}<x_{n, 2}<x_{n-2,2}<\cdots<x_{n, d_{n}-1}<x_{n-2, d_{n}-1}<x_{n, d_{n}}
$$

(ii) for every $n \geq 1$, the polynomial $W_{n}(z)$ has either one more (when $n$ is even) or the same number (when $n$ is odd) of roots as $W_{n-1}(z)$, and the roots interlace so that

$$
x_{n, 1}<x_{n-1,1}<x_{n, 2}<x_{n-1,2}<\cdots<x_{n-1, d_{n}-1}<x_{n, d_{n}} \text { when } n \text { even }
$$

and

$$
x_{n, 1}<x_{n-1,1}<x_{n, 2}<x_{n-1,2}<\cdots<x_{n, d_{n}}<x_{n-1, d_{n}} \text { when } n \text { odd } \text {. }
$$


Proof. From the initial polynomials (4.4), it is easy to verify Theorem 4.3 for $n \leq 2$. We suppose that $n \geq 3$ and proceed inductively.

For every $k \leq n-1$, we denote the roots of $W_{k}(z)$ by $x_{k, 1}<x_{k, 2}<\cdots<x_{k, d_{k}}$. For convenience, we define $x_{k, 0}=-\infty$ and $x_{k, d_{k}+1}=0$, for all $k \leq n-1$. To clarify the interlacing properties, we now consider the signs of the function $W_{m}(z)$ at $-\infty$ and at the origin, for any $m \geq 0$. Since $W_{m}(z)$ is a polynomial of degree $d_{m}$, with all coefficients non-negative, we deduce that

$$
(-1)^{d_{m}} W_{m}(-\infty)>0
$$

Having the constant term positive implies that

$$
W_{m}(0)=g_{n, 0}>0 .
$$

By the intermediate value theorem and Inequality (4.10), for the polynomial $W_{n}(z)$ to have $d_{n}=\operatorname{deg} W_{n}(z)$ distinct negative roots and for Part (i) of Theorem 4.3 to hold, it is necessary and sufficient that

$$
(-1)^{d_{n}+j} W_{n}\left(x_{n-2, j}\right)>0 \text { for } 1 \leq j \leq d_{n-2}+1 .
$$

In fact, for $j=d_{n-2}+1$, Inequality (4.12) becomes

$$
(-1)^{d_{n}+d_{n-2}+1} W_{n}(0)>0 .
$$

By (4.11), Inequality (4.13) holds if and only if $d_{n}+d_{n-2}$ is odd, which is true since

$$
d_{n}+d_{n-2}=\left\lceil\frac{n+1}{2}\right\rceil+\left\lceil\frac{n-1}{2}\right\rceil=2\left\lceil\frac{n-1}{2}\right\rceil+1
$$

Now consider any $j$ such that $1 \leq j \leq d_{n-2}$. We are going to prove (4.12). We will use the particular indicator function $\mathrm{I}_{\text {even }}$, which is defined by

$$
\mathrm{I}_{\text {even }}(n)= \begin{cases}1, & \text { if } n \text { is even, } \\ 0, & \text { if } n \text { is odd }\end{cases}
$$

Note that $x_{n-2, j}$ is a root of $W_{n-2}(z)$. By Recurrence (4.3), we have

$$
W_{n}\left(z_{n-2, j}\right)=x_{n-2, j}^{\mathrm{I}_{\text {even }}(n)}\left(20 W_{n-1}\left(x_{n-2, j}\right)-384 x_{n-2, j} W_{n-3}\left(x_{n-2, j}\right)\right) .
$$

Since $x_{n-2, j}<0$, the factor $x_{n-2, j}^{\mathrm{I}_{\text {even }}(n)}$ contributes $(-1)^{n+1}$ to the sign of the right hand side of (4.14). On the other hand, it is clear that the sign of the parenthesized factor can be determined if both the summands $20 W_{n-1}\left(x_{n-2, j}\right)$ and $-384 x_{n-2, j} W_{n-3}\left(x_{n-2, j}\right)$ have the same sign. Therefore, Inequality (4.12) holds if

$$
\begin{aligned}
& (-1)^{d_{n}+j+n+1} W_{n-1}\left(x_{n-2, j}\right)>0, \\
& (-1)^{d_{n}+j+n+1} W_{n-3}\left(x_{n-2, j}\right)>0 .
\end{aligned}
$$

By the induction hypothesis on part (ii) of this theorem, we can substitute $P=W_{n-1}$ and $Q=W_{n-2}$ into Lemma 4.2. Then Inequality (4.5) gives

$$
(-1)^{d_{n-1}+j} W_{n-1}\left(x_{n-2, j}\right)>0 .
$$


Thus, Inequality (4.15) holds if and only if the total power

$$
d_{n}+j+n+1+d_{n-1}+j=\left\lceil\frac{n+1}{2}\right\rceil+\left\lceil\frac{n}{2}\right\rceil+n+2 j+1
$$

of $(-1)$ in (4.15) and (4.17) is even, which is clear by a simple parity argument. Moreover, again using the induction hypothesis on part (ii), we can make substitutions $P(x)=$ $W_{n-2}(x)$ and $Q(x)=W_{n-3}(x)$ into Lemma 4.2. Then Inequality (4.6) gives

$$
(-1)^{d_{n-3}+j} W_{n-3}\left(x_{n-2, j}\right)<0 .
$$

Thus, Inequality (4.16) holds if and only if the total power

$$
d_{n}+j+n+1+d_{n-3}+j=\left\lceil\frac{n+1}{2}\right\rceil+\left\lceil\frac{n-2}{2}\right\rceil+n+2 j+1
$$

of $(-1)$ in (4.16) and (4.18) is odd, which is also clear by a simple parity argument. This completes the proof of (4.12), and the proof of Part (i).

The approach to proving Part (ii) is similar to that used to prove Part (i). By the intermediate value theorem and Inequality (4.10), Part (ii) holds if and only if

$$
(-1)^{d_{n}+j} W_{n}\left(x_{n-1, j}\right)>0 \text { for } 1 \leq j \leq d_{n-1},
$$

and also for $j=d_{n-1}+1$ when $n$ is even. In fact, when $n$ is even and $j=d_{n-1}+1$, we have

$$
(-1)^{d_{n}+d_{n-1}+1} W_{n}(0)>0 .
$$

By (4.11), Inequality (4.21) holds if and only if $(-1)^{d_{n}+d_{n-1}+1}=1$, which is clear since

$$
d_{n}+d_{n-1}+1=\left\lceil\frac{n+1}{2}\right\rceil+\left\lceil\frac{n}{2}\right\rceil+1=n+2 .
$$

For $1 \leq j \leq d_{n-1}$, we are now going to show (4.20). By setting $x=x_{n-1, j}$, Recurrence (4.3) turns into

$$
W_{n}\left(x_{n-1, j}\right)=8\left(3-8 x_{n-1, j}\right) W_{n-2}\left(x_{n-1, j}\right)-384 x_{n-1, j}^{1+\mathrm{I}_{\text {even }}(n)} W_{n-3}\left(x_{n-1, j}\right) .
$$

Since $x_{n-1, j}<0$, we see that $8\left(3-8 x_{n-1, j}\right)>0$, and that the factor $-384 x_{n-1, j}^{1+\mathrm{I}_{\text {even }}(n)}$ contributes $(-1)^{n+1}$ to the sign of the right-hand side of (4.22). Therefore, Inequality (4.20) holds if

$$
\begin{aligned}
(-1)^{d_{n}+j} W_{n-2}\left(x_{n-1, j}\right) & >0, \\
(-1)^{d_{n}+j+n+1} W_{n-3}\left(x_{n-1, j}\right) & >0 .
\end{aligned}
$$

Substituting $P(x)=W_{n-1}(x)$ and $Q(x)=W_{n-2}(x)$ into Lemma 4.2, we find that Inequality (4.6) yields

$$
(-1)^{d_{n-2}+j} W_{n-2}\left(x_{n-1, j}\right)<0 \quad \text { when } 1 \leq j \leq d_{n-1} .
$$

Thus, Inequality (4.23) holds if and only if the total power

$$
d_{n}+j+d_{n-2}+j=\left\lceil\frac{n+1}{2}\right\rceil+\left\lceil\frac{n-1}{2}\right\rceil+2 j
$$


of $(-1)$ in (4.23) and (4.25) is odd, which holds true, obviously, by parity. On the other hand, by the induction hypothesis on Part (i) and substituting $P(x)=W_{n-1}(x)$ and $Q(x)=W_{n-3}(x)$ into Lemma 4.2, Inequality (4.6) becomes

$$
(-1)^{d_{n-3}+j} W_{n-3}\left(x_{n-1, j}\right)<0 .
$$

Therefore, Inequality (4.24) holds if and only if the total power $d_{n}+j+n+1+d_{n-3}+j$ of $(-1)$ in (4.24) and (4.26) is odd, which coincides with (4.19). This completes the proof of (4.20), ergo the proof of Part (ii), and hence the entire theorem.

Corollary 4.4. The sequence of coefficients for every genus polynomial $\Gamma_{Y_{n}}(z)$ is logconcave.

Proof. Recalling Equation (4.1), we have

$$
\Gamma_{Y_{n}}(z)=z^{\lfloor(n+1) / 2\rfloor} W_{n}(z) .
$$

By Theorem 4.3, we know that the polynomial $W_{n}(z)$ is real-rooted. It follows that the polynomial $\Gamma_{Y_{n}}(z)$ is real-rooted. Applying Theorem 4.1 (Newton's theorem), we know that the polynomial $\Gamma_{Y_{n}}(z)$ is log-concave.

\section{On Real-Rootedness}

In the study of genus polynomials, the role of real-rootedness may rise beyond being a sufficient condition for log-concavity. The introductory section presents two basic research problems specifically on real-rootedness. One may reasonably anticipate that continuing study of the roots of genus polynomials will lead to new insights into the imbeddings of graphs.

\section{References}

[1] L. W. Beineke and R. J. Wilson (eds.), Topics in topological graph theory, volume 128 of Encyclopedia of Mathematics and its Applications, Cambridge University Press, Cambridge, 2009, doi:10.1017/CBO9781139087223, with academic consultants J.L. Gross and T.W. Tucker.

[2] M. L. Furst, J. L. Gross and R. Statman, Genus distributions for two first classes of graphs, J. Combin. Theory Ser. B 46 (1989), 22-36, doi:10.1016/0095-8956(89)90004-X.

[3] J. Gross, Embeddings of graphs of fixed treewidth and bounded degree, Ars Math. Contemp. 7 (2014), 379-403, presented at AMS Meeting in Boston, January 2012.

[4] J. L. Gross, Genus distribution of graph amalgamations: self-pasting at root-vertices, Australas. J. Combin. 49 (2011), 19-38.

[5] J. L. Gross, I. F. Khan and M. I. Poshni, Genus distribution of graph amalgamations: pasting at root-vertices, Ars Combin. 94 (2010), 33-53.

[6] J. L. Gross, I. F. Khan and M. I. Poshni, Genus distributions for iterated claws, Electron. J. Combin. 21 (2014), Paper 1.12, 21.

[7] J. L. Gross, T. Mansour, T. W. Tucker and D. G. L. Wang, Log-concavity of combinations of sequences and applications to genus distributions, SIAM J. Discrete Math. 29 (2015), 10021029, doi:10.1137/140978867. 
[8] J. L. Gross, D. P. Robbins and T. W. Tucker, Genus distributions for bouquets of circles, J. Combin. Theory Ser. B 47 (1989), 292-306, doi:10.1016/0095-8956(89)90030-0.

[9] J. L. Gross and T. W. Tucker, Topological graph theory, Dover Publications, Inc., Mineola, NY, 2001, reprint of the 1987 original [Wiley, New York; MR0898434 (88h:05034)] with a new preface and supplementary bibliography.

[10] L. L. Liu and Y. Wang, A unified approach to polynomial sequences with only real zeros, $A d v$. in Appl. Math. 38 (2007), 542-560, doi:10.1016/j.aam.2006.02.003.

[11] M. I. Poshni, I. F. Khan and J. L. Gross, Genus distributions of graphs under edgeamalgamations, Ars Math. Contemp. 3 (2010), 69-86.

[12] S. Stahl, On the zeros of some genus polynomials, Canad. J. Math. 49 (1997), 617-640, doi: 10.4153/CJM-1997-029-5.

[13] R. P. Stanley, Log-concave and unimodal sequences in algebra, combinatorics, and geometry, in: Graph theory and its applications: East and West (Jinan, 1986), New York Acad. Sci., New York, volume 576 of Ann. New York Acad. Sci., pp. 500-535, 1989, doi:10.1111/j.1749-6632. 1989.tb16434.x.

[14] D. Wagner, Zeros of genus polynomials of graphs in some linear families, Technical Report CORR 97-15, Univ. Waterloo, 1997, 9pp.

[15] H. S. Wilf, generatingfunctionology, A K Peters, Ltd., Wellesley, MA, 3rd edition, 2006. 